\title{
Optimising acute care and secondary prevention for patients with acute coronary syndrome
}

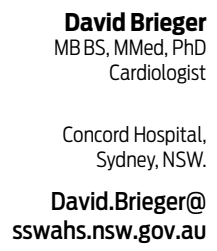

doi: 10.5694/mjal4.01249
P atients who experience an acute coronary syndrome (ACS) present with heterogeneous clinical manifestations. In the emergency department, risk assessment should immediately identify patients with ST-segment elevation myocardial infarction (STEMI) who are in need of emergency reperfusion. In patients with non-ST-segment elevation acute coronary syndrome (NSTEACS), the focus is on identifying those at higher risk of recurrent infarction or death. This then guides the application of evidence-based therapies such as angiography and appropriate revascularisation, powerful antithrombotic therapy and comprehensive secondary prevention.

Evaluations of clinical practice have consistently shown underuse of risk stratification and consequent inappropriate application of evidence-based practice. This is particularly true for patients at higher risk of adverse events, who are often undertreated yet have the most to gain from evidence-based therapy.

\section{Optimising systems of care for STEMI}

The acute occlusion of a major epicardial coronary vessel usually occurs in the context of poor collateral supply, and results in the rapid onset of myocardial necrosis accompanied by an increased likelihood of lethal arrhythmia. These patients have the highest inhospital mortality rates, but these can be improved by prompt institution of reperfusion therapy. There is a clear relationship between longer time from symptom onset to reperfusion and increasing mortality rates. SNAPSHOT ACS, the comprehensive binational audit run in Australia and New Zealand during 2 weeks of May 2012, ${ }^{1}$ reported median times from hospital presentation to reperfusion (measured as door-to-needle time) of 42 minutes (interquartile range, 25-70 minutes) for those receiving fibrinolysis, and 82 minutes (interquartile range, 53-138 minutes) for those receiving primary percutaneous coronary intervention (PCI; measured as door-to-balloon time). While these median intervals fall within recommended benchmark goals, an appreciable proportion of patients fell outside these goals of 60 minutes and 90 minutes for fibrinolysis and primary $\mathrm{PCI}$, respectively. More disturbingly, $32 \%$ of patients with confirmed STEMI presenting within 12 hours of the onset of symptoms did not receive any form of reperfusion therapy.

Upskilling the health care professionals who make first contact with patients, to allow early performance and interpretation of electrocardiograms (ECGs), can dramatically reduce the time from symptom development to reperfusion in patients with STEMI. This approach is being implemented in a variety of settings involving paramedics, general practitioners and nursing practitioners, with specialist support. Where the travel time

\begin{abstract}
Summary
Ascertaining a patient's risk of ischaemic complications after admission with an acute coronary syndrome is an important determinant of management.

The treating clinician must determine whether and how urgently to send the patient for coronary angiography and whether to select more intensive antithrombotic therapies in the acute phase, and facilitate secondary prevention strategies.

Risk stratification is infrequently applied and, as a consequence, undertreatment of higher-risk patients is common.

Ensuring routine application of risk stratification across hospitals may improve treatment of patients who have the most to gain from evidence-based therapies.

This requires embedding standard practices into complex clinical environments, and includes the routine implementation of treatment algorithms in a permissive environment with clinical champions and support from the hospital administration.

The implementation of routine systems of care defining prehospital, interhospital and individual hospital practice is challenging, but essential to minimise deficits in care.
\end{abstract}

to hospital is prolonged, fibrinolysis can be administered in the field. ${ }^{2}$ A recent Australian epidemiological modelling study found that this was the most effective way to optimise timely reperfusion for STEMI in remote locations. ${ }^{3}$ Elsewhere, the nearest PCI-capable hospital can be notified once the diagnosis is made, the cardiac catheterisation laboratory ("cath lab") activated, and the patient transported directly to the lab to meet the assembled team. 4

Hospitals without PCI facilities should have the capacity to efficiently transfer high-risk STEMI patients to a PCI-capable hospital, with or without first providing fibrinolysis. ${ }^{5}$ This transfer is most efficient if done direct from the emergency department, and requires an undertaking from the ambulance service that these patients are a priority, and a similar commitment from the receiving PCI-capable hospital to provide emergency access to the cath lab. Hospitals with PCI services can shorten times to reperfusion through the application of simple local practices, including emergency department activation of the cath lab, emergency department bypass when the diagnosis has been made in the field, and routine feedback of outcomes to all those involved in provision of this service. ${ }^{6,7}$

\section{Patients with non-ST-segment elevation ACS}

Patients presenting with NSTEACS are usually older than those with STEMI, with more extensive coronary disease. Their inhospital mortality varies appreciably, 
driven partly by the acute myocardial risk and partly by the presence of comorbidities. ${ }^{8}$ Outcomes can be improved by application of intensive evidence-based care, including antithrombotic medications, revascularisation where appropriate, and application of secondary prevention strategies. ${ }^{9}$ There is strong, consistent evidence that patients at the highest risk, with the most to gain from evidence-based therapies, are the least likely to receive it. ${ }^{10}$ It appears that while clinicians recognise the contribution of factors - such as advanced age or presenting in cardiac failure or after cardiac arrest - to an adverse prognosis, there may be a reluctance to subject higher-risk patients to the risks of procedures or side effects of therapies, and uncertainty around the applicability of evidence to populations poorly represented in randomised trials. ${ }^{11,12}$

When clinicians do assign evidence-based treatments, they use clinical assessments that focus on the presence of ECG changes or biomarker elevation. ${ }^{13}$ Objective riskstratification tools, which include other prognostic features including the presence of renal failure or advanced age, perform better than clinical assessment. ${ }^{14-16}$ These are available as mobile apps or on the internet, ${ }^{17,18}$ but are rarely used in practice. ${ }^{15}$

\section{Challenges to improving ACS care}

There is an association between application of evidencebased care and outcomes in patients with ACS; 19 however, strategies to systematically improve such care in Australia have proven disappointing. In the large Discharge Management of Acute Coronary Syndromes (DMACS) project, a strategy focused on academic detailing of hospital staff together with reminders and discharge tools improved prescription of evidence-based discharge therapies from $57 \%$ to $69 \%$. Important barriers to further improving uptake included high turnover of resident medical staff and senior clinician behaviour. The latter was characterised by a reluctance to accept some guideline recommendations and an unwillingness to change established practice. Despite the inclusion of strategies to improve communication of discharge plans to GPs, when patients were surveyed at 3 months, rates had fallen to $48 \%$ and $52 \%$, respectively. ${ }^{20}$ This suggests that additional factors conspire to erode continuation of evidence-based therapy after hospital discharge, and these are not affected by intensive inhospital interventions.

\section{Embedding routine risk stratification into clinical practice}

The earlier appropriate care is applied, the greater the likelihood of continued application of evidence-based care, and the better the outcomes. ${ }^{21,22}$ Because risk stratification should be one of the first objectives when assessing an ACS patient, it provides the earliest opportunity to define care. Application of a risk-stratification tool provides unambiguous definition of the patient at high risk, who has the most to gain from evidence-based care, and this strategy is recommended in local and international ACS guidelines. ${ }^{23,24}$ It is imperative that any risk tool be linked to treatment recommendations; the United Kingdom National Institute for Health and Care
Excellence guidelines provide graded recommendations for evidence-based care based on the Global Registry of Acute Coronary Events (GRACE) risk score. ${ }^{24}$ Despite the consistency of guideline recommendations, it is important to recognise that there is no interventional evidence to demonstrate that implementation of risk scores influences improvements in care and outcomes, although one study addressing this question is underway in Australia (Australian Clinical Trial Registration no. 12614000550606).

Changing care in complex clinical environments is challenging. There are several overarching principles that govern successful practice change. These include securing administrative support, identifying clinical champions and ensuring the new practice has minimal impact on workload.

\section{Opportunities for improving secondary prevention}

Secondary prevention, including application of evidencebased therapies, cardiac rehabilitation, and long-term risk factor control contributes to prevention of half of the deaths after acute myocardial infarction, yet is consistently underused. ${ }^{25,26}$ Patients undergoing revascularisation are more likely to receive appropriate secondary prevention than those who do not, despite the fact that the latter are at higher risk of subsequent events. ${ }^{27}$ Failure to revascularise appears to initiate a cycle of missed opportunity that can be corrected by early application of risk-assessment algorithms. These tools should contain treatment recommendations that define the therapies to be considered in hospital and those to be continued after discharge. Ensuring continuation of therapies after discharge remains challenging, and will require greater engagement of providers in the community committed to partnering with their patients to increase adherence to lifestyle improvement, risk factor control, and pharmacological therapies. ${ }^{28}$

Competing interests: No relevant disclosures.

Provenance: Commissioned; externally peer reviewed.

1 Chew DP, French J, Briffa TG, et al. Acute coronary syndrome care across Australia and New Zealand: the SNAPSHOT ACS study. Med J Aust 2013; 199: 185-191.

2 Danchin N, Durand E, Blanchard D. Pre-hospital thrombolysis in perspective. Eur Heart J 2008; 29: 2835-2842.

3 Ranasinghe I, Turnbull F, Tonkin A, et al. Comparative effectiveness of population interventions to improve access to reperfusion for ST-segmentelevation myocardial infarction in Australia. Circ Cardiovasc Qual Outcomes 2012; 5: 429-436.

4 Carstensen S, Nelson GC, Hansen PS, et al. Field triage to primary angioplasty combined with emergency department bypass reduces treatment delays and is associated with improved outcome. Eur Heart $J$ 2007; 28: 2313-2319.

5 Armstrong PW, Gershlick AH, Goldstein P, et al. Fibrinolysis or primary PCI in ST-segment elevation myocardial infarction. N Engl J Med 2013; 368: 1379-1387.

6 Bradley EH, Herrin J, Wang Y, et al. Strategies for reducing the door-toballoon time in acute myocardial infarction. N Engl J Med 2006; 355: 2308-2320.

7 Willson AB, Mountain D, Jeffers JM, et al. Door-to-balloon times are reduced in ST-elevation myocardial infarction by emergency physician activation of the cardiac catheterisation laboratory and immediate patient transfer. Med J Aust 2010; 193: 207-212.

8 Granger CB, Goldberg RJ, Dabbous O, et al. Predictors of hospital mortality in the Global Registry of Acute Coronary Events. Arch Intern Med 2003; 163: 2345-2353. 
9 Fox KA, Clayton TC, Damman P, et al. Long-term outcome of a routine versus selective invasive strategy in patients with non-ST-segment elevation acute coronary syndrome: a meta-analysis of individual patient data. J Am Coll Cardiol 2010; 55: 2435-2445.

10 Ranasinghe I, Alprandi-Costa B, Chow V, et al. Risk stratification in the setting of non-ST elevation acute coronary syndromes 1999-2007. Am J Cardiol 2011; 108: 617-624.

11 Califf RM, Peterson ED, Gibbons RJ, et al. Integrating quality into the cycle of therapeutic development. J Am Coll Cardiol 2002; 40: 1895-1901.

12 Chew DP, Juergens C, French J, et al. An examination of clinical intuition in risk assessment among acute coronary syndromes patients: observations from a prospective multi-center international observational registry. Int J Cardiol 2014; 171: 209-216.

13 Joynt KE, Huynh L, Amerena JV, et al. Impact of acute and chronic risk factors on use of evidence-based treatments in patients in Australia with acute coronary syndromes. Heart 2009; 95: 1442-1448.

14 Ramsay G, Podogrodzka M, McClure C, Fox KA. Risk prediction in patients presenting with suspected cardiac pain: the GRACE and TIMI risk scores versus clinical evaluation. QJM 2007; 100: 11-18.

15 Yan AT, Yan RT, Tan M, et al. Risk scores for risk stratification in acute coronary syndromes: useful but simpler is not necessarily better. Eur Heart J 2007; 28: 1072-1078.

16 Chew DP, Junbo G, Parsonage W, et al. Perceived risk of ischemic and bleeding events in acute coronary syndromes. Circ Cardiovasc Qual Outcomes 2013; 6: 299-308.

17 MDCalc. TIMI risk score for UA/NSTEMI. http://www.mdcalc.com/timirisk-score-for-uanstemi (accessed Sep 2014).

18 Global Registry of Acute Coronary Events. GRACE 2.0 risk calculator. http://www.gracescore.org/WebSite/default.aspx?ReturnUrl=\%2f (accessed Sep 2014).
19 Peterson ED, Roe MT, Mulgund J, et al. Association between hospital process performance and outcomes among patients with acute coronary syndromes. JAMA 2006; 295: 1912-1920.

20 Peterson GM, Thompson A, Pulver LK, et al. Management of acute coronary syndromes at hospital discharge: do targeted educational interventions improve practice quality? J Healthc Qual 2012; 34: 26-34.

21 Randomised trial of intravenous streptokinase, oral aspirin, both, or neither among 17,187 cases of suspected acute myocardial infarction: ISIS-2. ISIS-2 (Second International Study of Infarct Survival) Collaborative Group. Lancet 1988; 2: 349-360.

22 Weaver WD, Cerqueira M, Hallstrom AP, et al. Prehospital-initiated vs hospital-initiated thrombolytic therapy. The Myocardial Infarction Triage and Intervention Trial. JAMA 1993; 270: 1211-1216.

23 Chew DP, Aroney CN, Aylward PE, et al. 2011 Addendum to the National Heart Foundation of Australia/Cardiac Society of Australia and New Zealand Guidelines for the management of acute coronary syndromes (ACS) 2006. Heart Lung Circ 2011; 20: 487-502.

24 National Institute for Health and Care Excellence. Unstable angina and NSTEMI: the early management of unstable angina and non-ST-segmentelevation myocardial infarction. London: NICE, 2010. https://www.nice. org.uk/guidance/cg94/resources/guidance-unstable-angina-andnstemi-pdf (accessed Oct 2014).

25 Ford ES, Ajani UA, Croft JB, et al. Explaining the decrease in US deaths from coronary disease, 1980-2000. N Engl J Med 2007; 356: 2388-2398.

26 Brieger DB, Redfern J. Contemporary themes in acute coronary syndrome management: from acute illness to secondary prevention. Med J Aust 2013; 199: 174-178.

27 Wallentin L, Becker RC, Budaj A, et al. Ticagrelor versus clopidogrel in patients with acute coronary syndromes. N Engl J Med 2009; 361: 1045-1057.

28 Briffa T, Chow CK, Clark AM, Redfern J. Improving outcomes after acute coronary syndrome with rehabilitation and secondary prevention. Clin Ther 2013; 35: 1076-1081. 